\title{
Application and Mechanism of Inhalation Profile Improvement of DPI Formulations by Mechanofusion with Magnesium Stearate
}

\author{
Michiko Kumon, ${ }^{*, a}$ Satoshi Machida, ${ }^{b}$ Masahiko Suzuki, ${ }^{a}$ Akira Kusai, ${ }^{a}$ Etsuo Yonemochi, ${ }^{b}$ and \\ Katsuhide TERADA ${ }^{b}$ \\ ${ }^{a}$ Formulation Technology Research Laboratories, Daiichi Sankyo Co., Ltd.; 1-2-58 Hiromachi, Shinagawa-ku, Tokyo \\ 140-8710, Japan: and ${ }^{b}$ Faculty of Pharmaceutical Sciences, Toho University; 2-2-1 Miyama, Funabashi, Chiba \\ 274-8510, Japan. Received July 27, 2007; accepted March 3, 2008; published online March 5, 2008
}

\begin{abstract}
In our previous paper, we reported the inhalation properties of dry powder inhaler (DPI) formulations containing Compound $\mathrm{A}$ and mechanofusion-processed lactose carriers. The mechanofusion process with magnesium stearate (Mg-St) on the lactose carrier enhanced the fine particle fraction (FPF) value of the Andersen cascade impactor (ACI) study. The increase of FPF seemed to be associated with the increase of the dispersibility of drug particles. The objectives of this study were (1) to evaluate the applicability of lactose carrier mechanofusion-processed with Mg-St and (2) to examine the mechanism of FPF alteration by the mechanofusion process applied on the lactose carrier with or without additive. The inhalation profiles of DPI formulations containing four different pharmaceutical compounds were evaluated with an ACI. The dispersibility of the formulations was observed by particle size distribution measurement in the air stream and the adhesive force was measured by direct separation method. It was found that higher FPF was obtained with lactose mechanofusion-processed with $\mathrm{Mg-St}$ as compared to control lactose carriers for all four compounds. This suggested that mechanofusion process with Mg-St is widely applicable in DPI formulations. The homogenization of surface adhesiveness was attributed to the increased FPF of the DPI including lactose mechanofusion-processed with Mg-St, as suggested by the combination of several physicochemical characteristics. Combination of different characterization methods would be of help to clarify the whole mechanism which defines the inhalation properties of DPI formulations.
\end{abstract}

Key words dry powder inhaler; lactose; surface property; mechanofusion; magnesium stearate

Dry powder inhaler (DPI) formulations have been utilized in the treatment of respiratory disease ${ }^{1-3)}$ and for systemic administration. ${ }^{4-7)}$ They have become common in the inhalation therapy field because of various advantages, such as being free from anti-environmental propellants ${ }^{8)}$ and ease of use with portable small devices.

DPI formulations with fine drug particles and coarse carrier particles have been widely used for a long time. Usually their components are simple and they can be manufactured with conventional equipment. Therefore, they appear to be a valuable option as the formulation for clinical trials, especially in the early stages of development. The carrier particles play the critical role in preventing the agglomeration of fine drug particles which possesses high surface energy due to their large specific surface area. On the other hand, it is necessary to achieve the high deposition in the deep lungs to satisfy the required bioavailability. Because only small particles (aerodynamic diameter below $5 \mu \mathrm{m}$ ) can reach the lungs, fine drug particles should be detached from the coarse carrier particles through the inhalation process. The percentage of small particles to the entire dose is defined as fine particle fraction (FPF). Therefore, FPF is an index of the extent of deposition in the lungs.

Studies have been conducted to clarify the factors that control the inhalation properties of the carrier-based DPI formulations. ${ }^{9-14)}$ Many studies focused on the carrier surface properties especially rugosity and/or coverage of high energy binding sites. ${ }^{10,15-21)}$ Surface energy of particles would be one of the major factors influencing the inhalation profile of DPI including FPF. ${ }^{22,23)}$ However the mechanism of FPF increase has not been fully clarified so far. This seems to be because the inhalation profile of DPI is affected by various factors including particle size, morphology electrostatic charge and inhalation condition. ${ }^{10,12,24-26)}$ Therefore, there is a need for preparation and characterization of series of powder samples that are different in surface properties but similar in other properties and compare them under the same inhalation condition.

Mechanofusion can modify the surface energy of lactose carrier particles without drastic change of the particle size and can make the particle shape round to lessen the differences.

Inverse gas chromatography (IGC) has been utilized to examine the effect of surface energy on the inhalation behavior of DPI. We reported that mechanofusion on a lactose carrier changed the carrier's surface condition and the inhalation properties of DPI formulations containing the lactose carrier and Compound A. ${ }^{27)}$ The Andersen cascade impactor (ACI) profile suggested that the increase of FPF was accompanied by the decrease of the interaction between Compound $\mathrm{A}$ and carrier particles. Begat et al. also reported that mechanofusion of lactose with $\mathrm{Mg}$-St reduced the interaction between drug and lactose particles. ${ }^{28)} \mathrm{Mg}$-St is known as lubricant and utilized to modify DPI formulation properties such as moisture resistance ${ }^{29)}$ and particle-particle interaction. ${ }^{28)}$

On the other hand, mechanofusion enhanced the surface energy of the lactose carrier. As the surface energy enhancement could be reflected in an increase of the surface adhesiveness, ${ }^{30,31)}$ there seemed to be discrepancy between the ACI study profile and the measured surface adhesiveness.

In this study, the applicability of a mechanofusionprocessed carrier was evaluated using four pharmaceutical compounds. Then we discussed the mechanism of the inhalation profile change by a mechanofusion process with some additives, particularly focused on the surface adhesiveness. 
Table 1. Mechanofusion Processing Conditions of Carrier Lactose Used for Each Study

\begin{tabular}{|c|c|c|c|c|c|c|c|}
\hline \multirow[b]{2}{*}{ Lactose } & \multicolumn{3}{|c|}{ Mechanofusion condition } & \multicolumn{3}{|c|}{ Study } & \multirow{2}{*}{$\begin{array}{c}\text { BET Surface } \\
\text { area } \\
\left(\mathrm{m}^{2} / \mathrm{g}\right)\end{array}$} \\
\hline & Power & $\begin{array}{l}\text { Time } \\
(\mathrm{min})\end{array}$ & Additive & $\begin{array}{c}\text { ACI study } \\
\text { (Figs. 1, } 2 \text { and/or 3) }\end{array}$ & $\begin{array}{c}\text { Particle size distribution } \\
\text { measurement } \\
\text { (Figs. 4, 5) }\end{array}$ & $\begin{array}{l}\text { Adhesive force } \\
\text { measurement } \\
\text { (Fig. 6) }\end{array}$ & \\
\hline Lactohale 100 & - & - & - & $\bigcirc^{a)}$ & - & 0 & 0.1 \\
\hline Lactohale 100 & Weak & 15 & - & $\bigcirc^{a)}$ & - & 0 & 0.2 \\
\hline Lactohale 100 & Weak & 15 & Sucrose stearate $1 \%$ & $\bigcirc^{a)}$ & - & 0 & 0.2 \\
\hline Lactohale 100 & Weak & 15 & Mg-St 3\% & $\bigcirc^{a)}$ & - & $\bigcirc$ & 0.3 \\
\hline Pharmatose $325 \mathrm{M}$ (Intact) & - & - & - & $\bigcirc^{a, b, c)}$ & 0 & O & 0.2 \\
\hline Pharmatose $325 \mathrm{M}$ & Medium & 30 & - & $\bigcirc^{a)}$ & 0 & 0 & 0.3 \\
\hline Pharmatose $325 \mathrm{M}$ & Medium & 15 & Sucrose stearate $1 \%$ & $\bigcirc^{a, c)}$ & 0 & 0 & 0.1 \\
\hline Pharmatose $325 \mathrm{M}$ & Medium & 15 & $\mathrm{Mg}-\mathrm{St} 3 \%$ & $\bigcirc^{a, b, c)}$ & O & ○ & 0.8 \\
\hline
\end{tabular}

a) Applied to the study for Fig. 1, b) applied to the study for Fig. 2,c) applied to the study for Fig. 3 .

\section{Experimental}

Materials Two grades of $\alpha$-lactose monohydrates, Lactohale $100(150$ $\mu \mathrm{m}$ in diameter) and Pharmatose $325 \mathrm{M}(60 \mu \mathrm{m}$ in diameter) were purchased from respectively Friesland Foods Domo (The Netherlands) and DMV (The Netherlands). Mg-St was purchased from Taihei Chemical Industrial Co., Ltd. (Japan) and sucrose stearate (S370F) was purchased from MitsubishiKagaku Foods Corporation (Japan). Dipalmitoylphosphatidylcholine was purchased from Wako Pure Chemical Industries, Ltd. (Japan). Triamcinolone acetonide (TAA) was supplied by Toho University (Japan) and disodium cromoglycate (cromolyn) was obtained from Sigma Aldrich Co. (U.S.A.). Compound A and 1-\{2-[(2R)-2-(3,4-dichlorophenyl)-4-(3,4,5trimethoxybenzoyl)morpholin-2-yl] ethyl $\}$ spiro[benzo[c]thiophene-1 $(3 H), 4^{\prime}$ piperidine]-(2S)-oxide hydrochloride (triple neurokinin receptors antagonist: TNRA) were synthesized by Daiichi Sankyo Co., Ltd. (Japan). Coomassie Brilliant Blue (R-250) (CBB) was purchased from Wako Pure Chemical Industries, Ltd. (Tokyo, Japan). CBB was sieved with a 350 mesh sieve before mixing with lactose.

Preparation of Lactose Carriers For the surface modification, lactose products (Lactohale 100 and Pharmatose $325 \mathrm{M}$ ) were treated by mechanofusion with or without additive using a rotor-type powder mixer, Mechanofusion ${ }^{\circledR}$ AMS (Hosokawa Micron Corporation, Japan). Briefly, the mechanofusion process was conducted with a Mechanofusion ${ }^{\circledR}$ AMS and the basic procedures were reported. ${ }^{27)}$ The detailed conditions of the mechanofusion process are shown in Table 1.

Preparation of Powder Formulation Drug compounds were milled with a Jet Mill Co-Jet system (Seishin Enterprise Co., Ltd., Japan) to the size of $2-3 \mu \mathrm{m}$ in diameter (Table 2). The modified lactose carrier and the milled drug particles were gently blended with a mortar and pestle at the ratios of $98: 2$ and $96: 4, w / w$. In the case of the ternary mixture of the milled drug, the lactose carrier and additive, the drug was added to the mixture of lactose and additive. After mixing, the mixture was sieved with a 60 mesh sieve. The drug concentration of each mixture thus obtained was in the range of 90 to $110 \%$ of that calculated from the feed composition. Twenty-five milligrams $( \pm 1 \mathrm{mg})$ of each mixture, drug powder or lactose carrier was loaded into HPMC capsules (size 2, Qualicaps Co., Ltd., Japan).

Physicochemical Characterization of Drug Particles, Carrier Lactose and Dry Powder Formulations The particle size distribution of the milled drug compounds, lactose carriers and DPI formulations were measured with a laser diffraction particle size distribution analyzer (Helos \& Rodos, Sympatec $\mathrm{GmbH}$, Germany). The specific surface area of the carrier lactose was measured by the BET adsorption method with nitrogen gas.

Cascade Impactor Test The inhalation properties of the DPI formulations were evaluated using an Andersen Cascade Impactor (ACI, Copley, U.K.) with an inhalation device, a Jethaler (dual chamber type, Hitachi Unisia Automotive, Ltd., Japan) in the manner as described for the DPI formulations containing Compound $\mathrm{A}^{27)}$ In brief, the formulated powder was filled in a capsule and inhaled with Jethaler at the flow rate of $301 / \mathrm{min}$. The amount of the drug deposited on each part of the ACI was measured by HPLC or UV absorption analysis. Lactose deposition was evaluated by weighing each part of ACI before and after the inhalation.

The fine particle fraction (FPF) is defined as the percentage of powder collected from Stage 2 to Stage 7 and a filter at $301 / \mathrm{min}$. FPF is given by Eq. 1:
Table 2. Surface Energy and Particle Sizes of Drug Compounds

\begin{tabular}{lcccc}
\hline \hline & & \multicolumn{3}{c}{ Particle size $(\mu \mathrm{m})$} \\
\cline { 3 - 5 } & $\gamma_{\mathrm{s}}^{\mathrm{D}}\left(\mathrm{mJ} / \mathrm{m}^{2}\right)$ & $\mathrm{D}_{10 \%}$ & $\mathrm{D}_{50 \%}$ & $\mathrm{D}_{90 \%}$ \\
\hline Compound A & $52.0(1.1)$ & $0.9(0.2)$ & $2.4(0.5)$ & $6.4(2.0)$ \\
TNRA & $66.3(1.9)$ & $0.7(0.1)$ & $1.8(0.4)$ & $4.4(0.6)$ \\
Cromolyn & $86.7(1.0)$ & $0.8(0.0)$ & $2.3(0.1)$ & $4.9(0.1)$ \\
TAA & $84.8(0.6)$ & $1.2(0.1)$ & $3.3(0.1)$ & $9.2(0.4)$ \\
\hline
\end{tabular}

Data represented as mean (S.D.) $n=3$.

$$
\begin{aligned}
\text { FPF }(\%)= & (\text { drug compound collected from Stage } 2 \text { to Stage } 7 \text { and } \\
& \text { a filter }) /(\text { entire dose }) \times 100
\end{aligned}
$$

Drug Analysis Compound A, TNRA and Cromolyn were analyzed by HPLC with a basic system consisting of a pump (Shimadzu Corporation, Japan) and a UV detector (Shimadzu Corporation, Japan). Regarding the analysis of Compound A, the details have already been reported. ${ }^{27)}$ TNRA was analyzed with an HPLC system employing a mixture of acetonitrile and $0.01 \mathrm{~mol} / 1$ sodium phosphate buffer $(\mathrm{pH} 7.0)(43: 57 \%, \mathrm{v} / \mathrm{v})$ as the mobile phase running at a flow rate of $c a .1 \mathrm{ml} / \mathrm{min}$ and UV detection at $254 \mathrm{~nm}$. The ODS column (L-column ODS, $15 \mathrm{~cm} \times 4.6 \mathrm{~mm}$ i.d., particle size $5 \mu \mathrm{m}$, Chemicals Evaluation and Research Institute, Japan) was maintained at $40^{\circ} \mathrm{C}$. Cromolyn was analyzed with an HPLC system employing a mixture of acetonitrile and $0.1 \%(\mathrm{v} / \mathrm{v})$ phosphoric acid $(\mathrm{pH} 3.0)(30: 70 \%$, v/v) containing $0.05 \%$ tetra- $n$-butyl ammonium bromide as an ion pair agent as the mobile phase running at a flow rate of $c a .1 \mathrm{ml} / \mathrm{min}$ and UV detection at $240 \mathrm{~nm}$. The same column conditions as those for TNRA were used. TAA was analyzed by UV absorption at $254 \mathrm{~nm}$ with a UV-spectrophotometer (UV-1600PC, Shimadzu Corporation, Japan).

Measurement of Surface Property with Inverse Gas Chromatography (IGC) Experiments were performed using an IGC (Surface Measurement Systems Ltd., U.K.). The basic method has already been reported elsewhere. ${ }^{27)}$ Briefly, samples were packed into a silanised glass column. Methane was used for the inert reference; $n$-decane, $n$-nonane, $n$-octane and $n$-heptane were used to determine the alkane line. Powder surface energy can be calculated from the retention time of nonpolar probes. The methodology utilized is described by Shultz et al. ${ }^{32)}$ As Grimsey et al. reported, ${ }^{33)}$ the basic relationship employed is:

$$
R T \ln V_{\mathrm{n}}=2 N\left(\gamma_{\mathrm{s}}^{\mathrm{D}}\right)^{1 / 2} a\left(\gamma_{1}^{\mathrm{D}}\right)^{1 / 2}+C
$$

where $R$ is the gas constant, $T$ is the temperature $(\mathrm{K}), V_{\mathrm{n}}$ is the net retention volume of the probe, $N$ is Avogadro's number, $a$ is the molecular surface area of the probe, $\gamma_{\mathrm{s}}^{\mathrm{D}}$ is the dispersive component of the surface energy of the sample powder, $\gamma_{1}^{\mathrm{D}}$ is the dispersive component of the surface energy of the probe and $C$ is a constant. Plotting $R T \ln V_{\mathrm{n}} v s . a\left(\gamma_{1}^{\mathrm{D}}\right)^{1 / 2}$ for the nonpolar probes yields a straight line (alkane line). The dispersive component of the solids is calculated from the slope in Eq. 2. The value of a and $\gamma_{1}^{\mathrm{D}}$ were obtained from the literature. ${ }^{32,34)}$

Measurement of Adhesive Force The adhesive force between the drug particle and the lactose carrier particle was measured by the direct separa- 
Table 3. Effect of Dispersive Component of Lactose Surface Energy on Fine Particle Fraction

[Base lactose: Pharmatose 325M]

\begin{tabular}{|c|c|c|c|c|c|c|c|c|}
\hline \multirow{3}{*}{\multicolumn{2}{|c|}{ Lactose carrier }} & \multirow{3}{*}{$\begin{array}{c}\gamma_{\mathrm{s}}^{\mathrm{D}} \text { of } \\
\text { lactose } \\
\left(\mathrm{mJ} / \mathrm{m}^{2}\right)\end{array}$} & \multicolumn{6}{|c|}{ FPF (\%) } \\
\hline & & & \multicolumn{2}{|c|}{ Compound A } & \multicolumn{2}{|c|}{ TNRA } & \multirow{2}{*}{$\begin{array}{c}\text { Cromolyn } \\
2 \%\end{array}$} & \multirow{2}{*}{$\begin{array}{c}\text { TAA } \\
4 \%\end{array}$} \\
\hline & & & $2 \%$ & $4 \%$ & $2 \%$ & $4 \%$ & & \\
\hline \multicolumn{2}{|c|}{ Intact (Pharmatose 325M) } & $46.9(2.6)$ & $20.8(1.8)$ & $25.1(3.1)$ & $28.1(0.4)$ & $20.5(6.3)$ & $31.7(2.8)$ & $32.5(1.0)$ \\
\hline $\begin{array}{l}\text { Mechanofusion } \\
\text { processed }\end{array}$ & $\begin{array}{l}\text { Without additive } \\
+ \text { Sucrose stearate } \\
+\mathrm{Mg}-\mathrm{St}\end{array}$ & $\begin{array}{l}50.6(7.2) \\
64.9(0.8) \\
76.3(1.5)\end{array}$ & $\begin{array}{l}13.2(0.3)^{* *} \\
25.7(2.5)^{*} \\
42.4(1.3)^{* *}\end{array}$ & $\begin{array}{l}20.4(3.7) \\
35.2(0.3)^{* *} \\
42.7(3.2)^{* *}\end{array}$ & $\begin{array}{l}11.7(3.4)^{* *} \\
20.9(2.4)^{* *} \\
34.6(2.3)^{* *}\end{array}$ & $\begin{array}{l}19.8(2.1) \\
27.5(2.1) \\
30.2(0.0)^{*}\end{array}$ & $\begin{array}{l}27.6(3.2) \\
29.7(1.6) \\
42.0(1.1)^{* *}\end{array}$ & $\begin{array}{l}22.4(0.2)^{* *} \\
26.9(1.0)^{* *} \\
38.8(2.2)^{*}\end{array}$ \\
\hline $\begin{array}{l}\text { Simple mixing } \\
\text { with mortar } \\
\text { and pestle }\end{array}$ & $\begin{array}{l}+ \text { Sucrose stearate } \\
+\mathrm{Mg}-\mathrm{St}\end{array}$ & $\begin{array}{l}47.7(0.9) \\
40.7(0.6)\end{array}$ & $\begin{array}{l}\text { NT } \\
\text { NT }\end{array}$ & $\begin{array}{l}\text { NT } \\
\text { NT }\end{array}$ & $\begin{array}{l}\text { NT } \\
\text { NT }\end{array}$ & $\begin{array}{l}12.4(1.0)^{* *} \\
14.7(1.0)^{* *}\end{array}$ & $\begin{array}{l}\text { NT } \\
\text { NT }\end{array}$ & $\begin{array}{l}\text { NT } \\
\text { NT }\end{array}$ \\
\hline
\end{tabular}

[Base lactose: Lactohale 100]

\begin{tabular}{|c|c|c|c|c|c|c|c|c|}
\hline \multirow{3}{*}{\multicolumn{2}{|c|}{ Lactose carrier }} & \multirow{3}{*}{$\begin{array}{c}\gamma_{\mathrm{s}}^{\mathrm{D}} \text { of } \\
\text { lactose } \\
\left(\mathrm{mJ} / \mathrm{m}^{2}\right)\end{array}$} & \multicolumn{6}{|c|}{ FPF $(\%)$} \\
\hline & & & \multicolumn{2}{|c|}{ Compound A } & \multicolumn{2}{|c|}{ TNRA } & \multirow{2}{*}{$\begin{array}{c}\text { Cromolyn } \\
2 \%\end{array}$} & \multirow{2}{*}{$\begin{array}{c}\text { TAA } \\
4 \%\end{array}$} \\
\hline & & & $2 \%$ & $4 \%$ & $2 \%$ & $4 \%$ & & \\
\hline \multicolumn{2}{|c|}{ Intact (Lactohale 100) } & $38.5(0.1)$ & $28.9(1.2)$ & $34.8(1.3)$ & NT & $13.7(1.3)$ & $33.2(0.3)$ & $32.7(2.4)$ \\
\hline $\begin{array}{l}\text { Mechanofusion } \\
\text { processed }\end{array}$ & $\begin{array}{l}\text { Without additive } \\
+ \text { Sucrose stearate } \\
+\mathrm{Mg}-\mathrm{St}\end{array}$ & $\begin{array}{l}43.8(0.2) \\
56.2(0.9) \\
60.8(1.3)\end{array}$ & $\begin{array}{l}25.8(1.3)^{*} \\
32.5(0.9)^{*} \\
39.3(1.9)^{* *}\end{array}$ & $\begin{array}{l}25.8(1.1)^{* *} \\
33.3(0.4) \\
42.4(2.1)^{* *}\end{array}$ & $\begin{array}{l}\text { NT } \\
\text { NT } \\
\text { NT }\end{array}$ & $\begin{array}{l}25.4(0.5)^{* *} \\
26.5(1.8)^{* *} \\
34.1(5.1)^{* *}\end{array}$ & $\begin{array}{l}27.3(0.3)^{* *} \\
31.9(2.1) \\
40.5(3.5)^{*}\end{array}$ & $\begin{array}{l}28.0(3.3) \\
31.7(4.9) \\
38.7(1.7)^{*}\end{array}$ \\
\hline
\end{tabular}

The surface energy of intact and mechanofusion-processed Pharmatose $325 \mathrm{M}$ and Lactohale 100 were reported earlier. ${ }^{27)}$ Data represented as mean (S.D.) $n=3$. $* p<0.05$, ** $p<0.01$, significant difference compared to intact lactose by Student's unpaired $t$-test. $\quad \mathrm{NT}=$ Not tested.

tion method using PAF-300N (OKADA SEIKO Co., Ltd., Japan). A lactose particle was attached to the end of the contact needle and drug particles were attached to the stage plate. The particles were attached to each other by moving the stage to the needle. After the attachment, the stage was moved away from the needle at a speed of about $0.6 \mu \mathrm{m} / \mathrm{s}$. When the particles were separated, the displacement of the contact needle was measured and the adhesive force was calculated based on the spring constant. The basic theory and details of this calculation are reported elsewhere. ${ }^{35,36)}$

Microscopic Observation of Drug Particles on Carrier Lactose Surface A powder of $\mathrm{CBB}$ was employed as a model drug powder. The carrier lactose and CBB were mixed in the same manner as described in 'Preparation of Powder Formulation.' The powder was observed with a digital microscope VH-Z450 (VH-8000C) (KEYENCE CORPORATION, Japan). To obtain a view of the overall-in-focus image, photographs of the target at different focus depths were taken. From these, photographs of different focus depths were composed and an overall-in-focus image of the target was obtained.

\section{Results}

The Inhalation Profile of Andersen Cascade Impactor The DPI formulations of the four pharmaceutical compounds mixed with lactose carriers were prepared and their inhalation profiles were evaluated with ACI (Fig. 1, Table 3). Data of Compound A in Fig. 1 and Table 3 were cited from our previous report. ${ }^{27)}$ For all four drugs, the lactose mechanofusion-processed with Mg-St showed higher FPF compared to the intact lactose. Namely, mechanofusion with $\mathrm{Mg}-\mathrm{St}$ decreased the deposition of drugs on the upper part, especially on the pre-separator, and increased it on the lower stages compared to intact lactose (Fig. 1). In contrast, mechanofusion without additives showed the tendency of decreasing FPF except the case of TNRA with Lactohale 100. Mechanofusion process with sucrose stearate showed the similar alteration of ACI deposition profile to that with Mg-St, but not as distinct as it was. Generally, the trend of the inhalation profile change was kept over the two different lactose particle sizes of 60 and $150 \mu \mathrm{m}$ (Figs. 1a-d and $\mathrm{e}-\mathrm{h}$ ). In the comparison with other testing conditions (Figs. $\mathrm{lb}-\mathrm{h}$ ), Compound A showed the different deposition pattern depending on the mechanofusion condition most clearly, especially with Pharmatose $325 \mathrm{M}$ (Fig. 1a). And there were some exceptions as follows: TNRA with intact Lactohale 100 showed relatively high deposition on capsules and devices and resulted in a lower FPF (Fig. 1f) compared with Pharmatose $325 \mathrm{M}$. Cromolyn with Pharmatose $325 \mathrm{M}$ mechanofusion-processed without additive showed lower deposition on pre-separator compared to the other drug compounds and resulted in relatively high FPF (Fig. 1c). TAA showed the smaller difference among the ACI deposition profile of 4 different formulations (Figs. 1d, h).

To investigate the effect of the incorporation of the carrier into the DPI formulations, the ACI deposition patterns of carrier lactose and drug compounds with or without carrier were compared by using Compound A (Fig. 2a) and TNRA (Fig. 2b). As lactose carrier itself was mainly deposited on the pre-separator, the drug particles needed to be detached from the carrier to be deposited as FPF. When Compound A was inhaled without carrier, only small FPF was obtained and the deposition on the pre-separator was increased (Fig. 2a). This suggests that highly cohesive fine drug particles formed agglomerates which is not redispersible. As well, TNRA without lactose showed small FPF and the majority of the drug remained in the capsule (Fig. 2b). Indeed, large ag- 
(a)

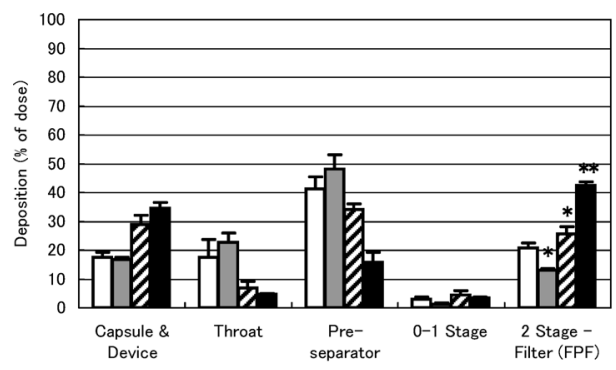

(b)

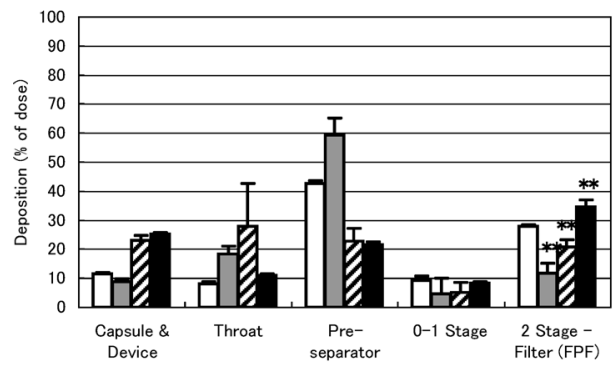

(c)

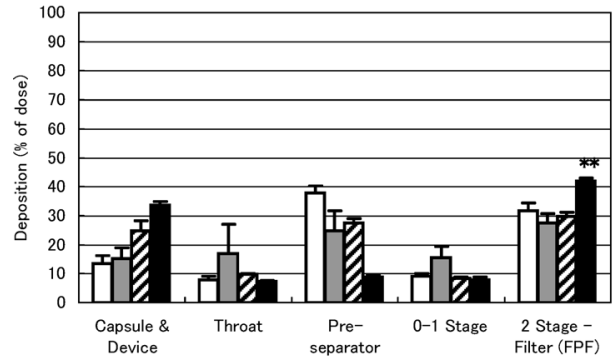

(d)

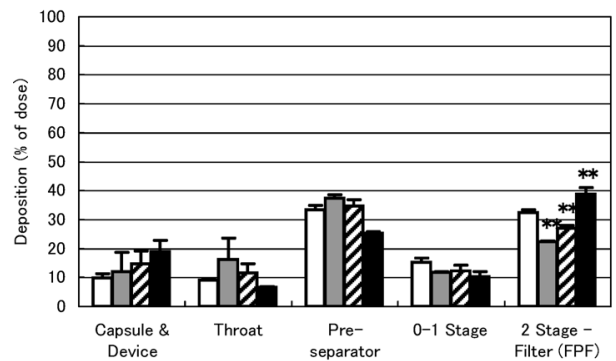

(e)

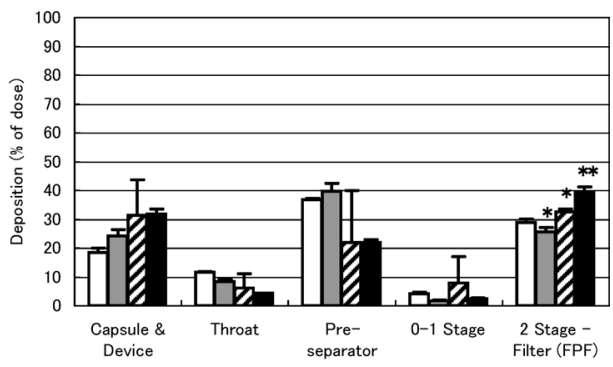

(f)

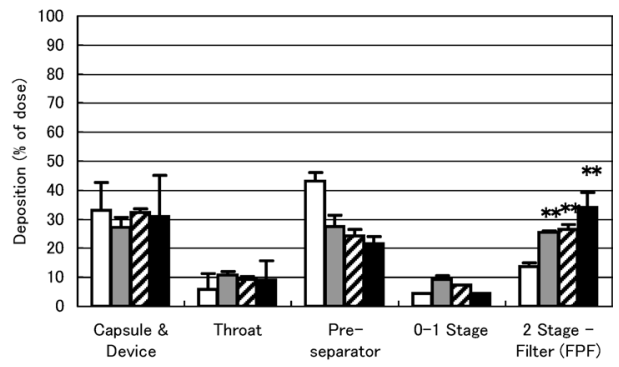

(g)

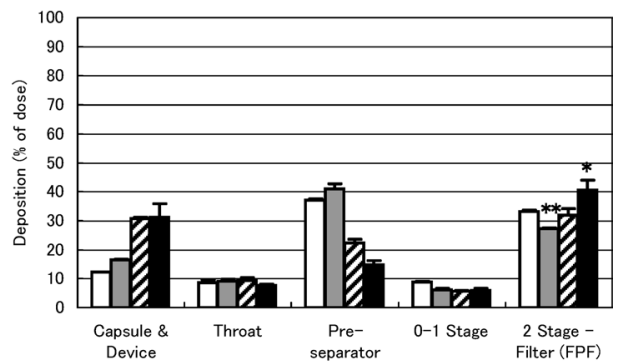

(h)

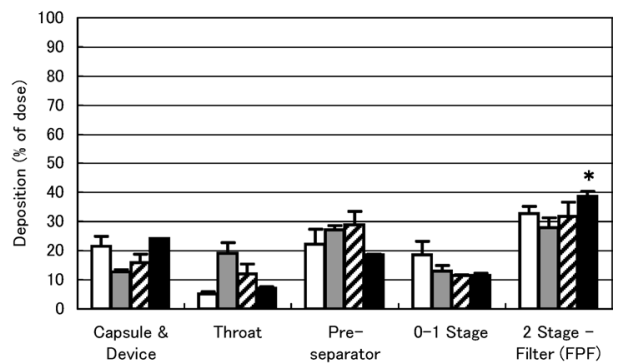

$\square$ Intact lactose

$\square$ Lactose mechanofusion-processed without additive

- Lactose mechanofusion-processed with sucrose stearate

- Lactose mechanofusion-processed with Mg-St

Fig. 1. Cascade Impactor Deposition Profile of DPI Formulations Containing Mechanofusion-Processed Lactose

(a) Compound A was mixed with Pharmatose 325M or mechanofusion-processed Pharmatose 325M at the ratio of 2:98 (w/w). (b) TNRA was mixed with Pharmatose 325M or mechanofusion-processed Pharmatose $325 \mathrm{M}$ at the ratio of $2: 98(\mathrm{w} / \mathrm{w})$. (c) Cromolyn was mixed with Pharmatose $325 \mathrm{M}$ or mechanofusion-processed Pharmatose $325 \mathrm{M}$ at the ratio of $2: 98(\mathrm{w} / \mathrm{w})$. (d) TAA was mixed with Pharmatose $325 \mathrm{M}$ or mechanofusion-processed Pharmatose $325 \mathrm{M}$ at the ratio of $4: 96$ (w/w). (e) Compound A was mixed with Lactohale 100 or mechanofusion-processed Lactohale 100 at the ratio of 2:98 (w/w). (f) TNRA was mixed with Lactohale 100 or mechanofusion-processed Lactohale 100 at the ratio of $4: 96(\mathrm{w} / \mathrm{w})$. (g) Cromolyn was mixed with Lactohale 100 or mechanofusion-processed Lactohale 100 at the ratio of $2: 98(\mathrm{w} / \mathrm{w})$. (h) TAA was mixed with Lactohale 100 or mechanofusion-processed Lactohale 100 at the ratio of $4: 96(\mathrm{w} / \mathrm{w})$. Error bars denote standard deviation, $n=3$. $* p<0.05$, $* * p<0.01$, significant difference compared to intact lactose by Student's unpaired $t$-test. 
(a)

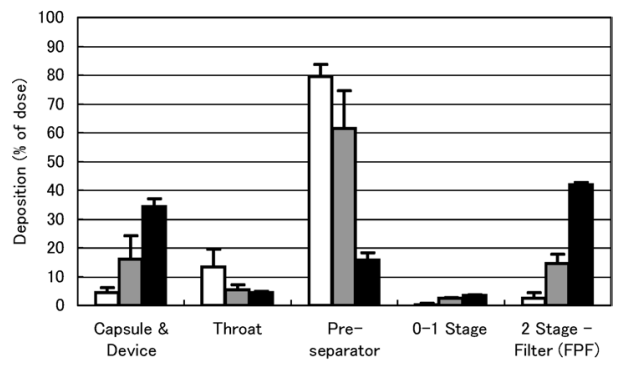

(b)

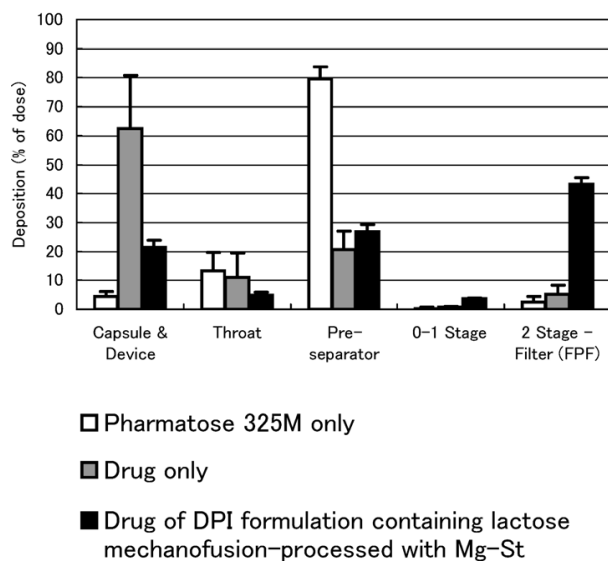

Fig. 2. ACI Deposition Profile of Lactose (Pharmatose 325M) Only, Drug Particles Only and DPI Formulation Containing Drug Particles

DPI formulations were composed of drug and Pharmatose $325 \mathrm{M}$ mechanofusionprocessed with $\mathrm{Mg}$-St $(2: 98)$. The drug particles used for the study were compound A (a) and TNRA (b). Error bars denote standard deviation, $n=3$.

glomerates were observed in the capsule after inhalation.

Influence of BET surface area on FPF value was evaluated and there was no clear correlation between them. DPI formulations containing lactose mechanofusion-processed with $\mathrm{Mg}$-St showed higher FPF than other carrier lactose over a wide range of surface area.

The effect of mechanofusion process was evaluated through the comparison between the simple mixing and mechanofusion process (Fig. 3). Simple mixing of lactose and additive was not as effective as the mechanofusion of additive on the lactose surface to achieve a high deposition on the lower stages of the ACI. This indicated that the mechanofusion process plays an important role in demonstrating high FPF values in DPI formulation containing additives.

Evaluation of Detachment of Drug Particles from Carrier in the Air Stream The particle size distributions of the lactose carriers and DPI formulations were evaluated. As shown in Fig. 4, in the case when measurement was performed under the dispersion pressure of $0.1 \mathrm{bar}$, larger amount of small particles was detected in DPI than in carrier lactose powder. The increased amount of small particles was considered to be due to the increased fine drug particles detached from the carrier lactose by the dispersion pressure. Therefore, the subtraction of the particle ratio of the carrier lactose from that of the DPI formulation at each particle size should be reflected the dispersibility of the drug particles $(\Delta$ accumulative \%). $\Delta$ accumulative $\%$ at the dispersing pressure of 0.1 bar (Fig. 5a) shows that drug particles was dis- (a)

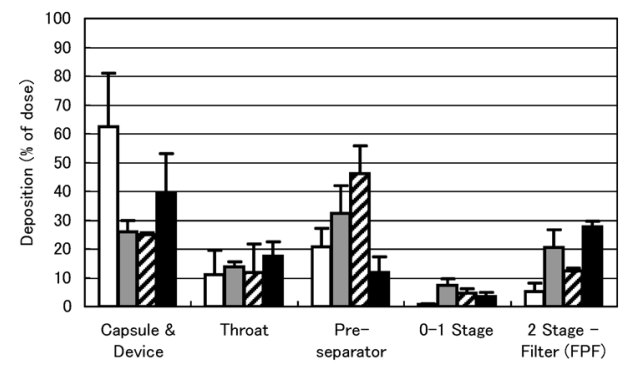

(b)

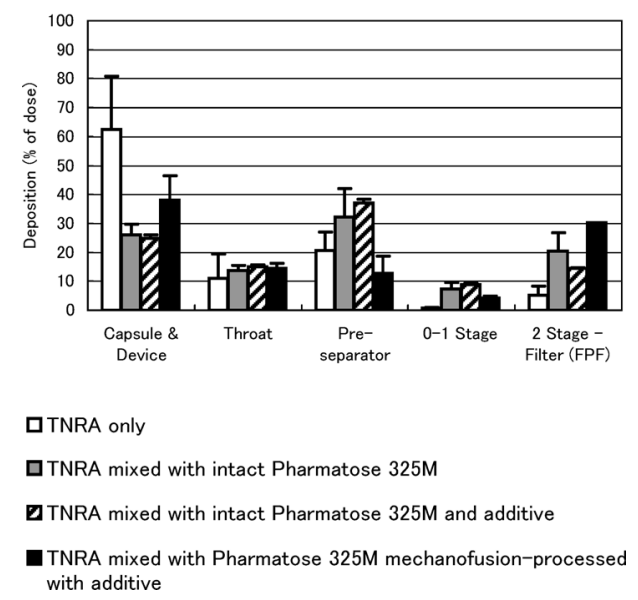

Fig. 3. ACI Deposition Profile of TNRA of DPI Formulations Containing Lactose and Additive or Lactose Mechanofusion-Processed with Additive

TNRA concentration of DPI formulation was $4 \%$. Additive was sucrose stearate (a) or Mg-St (b).

persed more easily when they were formulated with lactose mechanofusion-processed with additives than with intact lactose. As the dispersive pressure increased, the amount of small particles was increased for all lactoses, and the difference among them became unclear (Fig. 5).

Evaluation of Surface Energy by IGC The surface energy of the drug compounds, intact lactose, mechanofusionprocessed lactose and the mixture of the intact lactose and the additives was measured and the results are shown in Tables 2 and 3. The surface energy of drug particles ranged from 52.0 to $86.7 \mathrm{~mJ} / \mathrm{m}^{2}$. In mechanofusion-processed powder, $\mathrm{Mg}$-St and sucrose stearate increased the surface energy, whereas the simple mixing of lactose and additive without mechanical compression did not increase the surface energy (Table 3). This indicated that mechanofusion process in the presence of additives drastically changed surface property of the particles, namely increased surface energy.

Adhesive Force between Carrier Lactose and Drug Particles Direct measurement of the adhesive force between the carrier lactose and the drug particles was conducted and the results are shown in Fig. 6. The measurements were repeated 25 times for each sample. When the adhesive force was within the measurable range, the carrier lactose and drug particles were estimated to be separated. In contrast, when the adhesive force was over the measurement range, the drug particle was separated from the plate while it was attaching to the carrier lactose. Thus, the adhesive force was considered to exceed the measurable range. The upper 
(a)

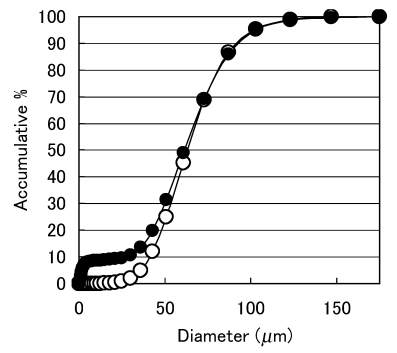

(e)

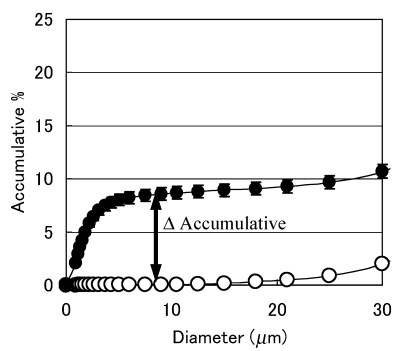

(b)
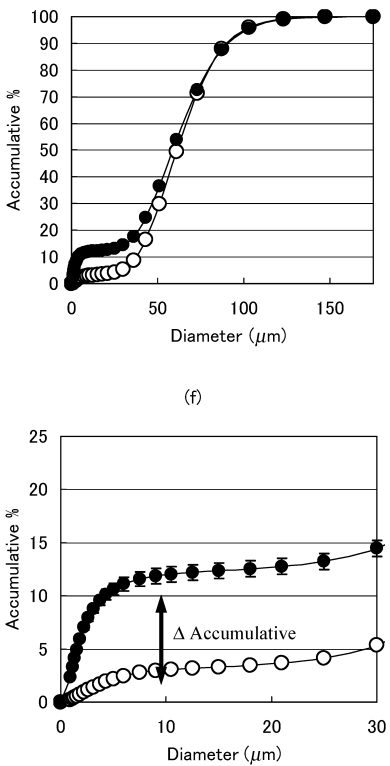

(c)

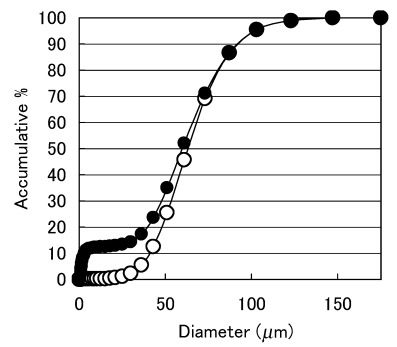

(g)

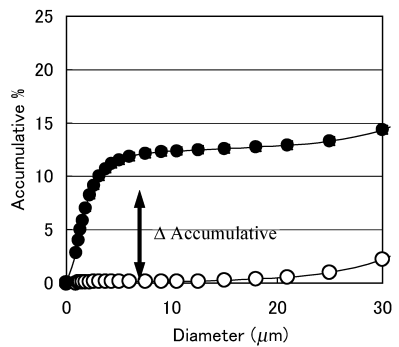

(d)
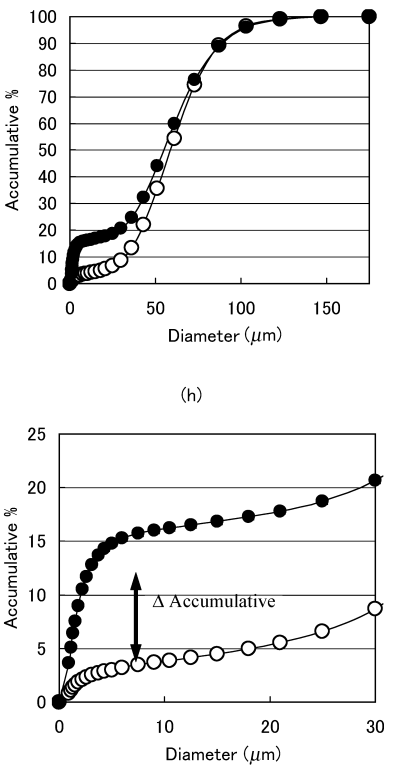

Fig. 4. Particle Size Distribution of Carrier Lactose and DPI Formulations Containing Lactose and Additive or Lactose Mechanofusion-Processed with Additive

O: carrier lactose, 0 : DPI formulation of TNRA 4\%. Lactose was intact (a), mechanofusion-processed without additive (b), mechanofusion-processed with sucrose stearate (c) and mechanofusion-processed with Mg-St (d). (e), (f), (g), (h) are the magnification of (a), (b), (c), (d) respectively. Dispersion pressure was 0.1 bar.
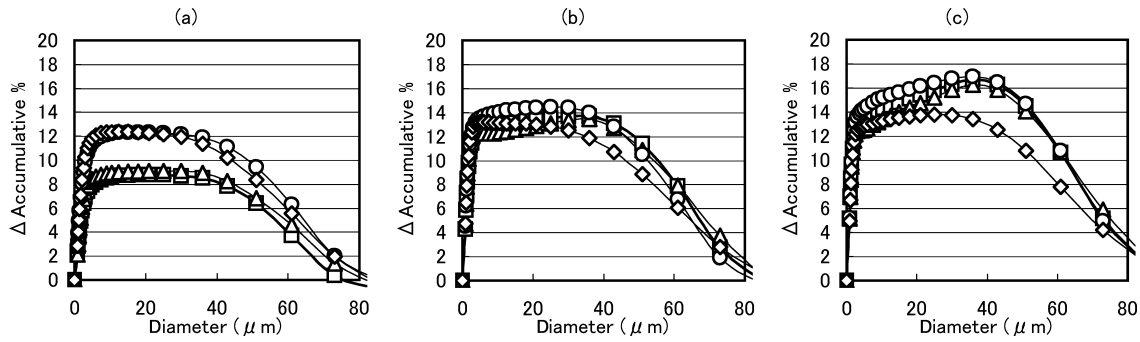

Fig. 5. Detachment of Drug Particles from Carrier Evaluated by the Comparison of Particle Size Distribution of $\Delta$ Accumulative $\%$ Profile of TNRA $4 \%$ DPI Dispersed at 0.1 bar (a), 2.0 bar (b), 4.0 bar (c)

$\square$ : Intact Pharmatose $325 \mathrm{M}, \triangle$ : Pharmatose $325 \mathrm{M}$ mechanofusion-processed without additive, $\bigcirc$ : Pharmatose $325 \mathrm{M}$ mechanofusion-processed with sucrose stearate, $\diamond$ : Pharmatose $325 \mathrm{M}$ mechanofusion-processed with $\mathrm{Mg}$-St.

limit is shown by dotted lines in Fig. 6. The frequency of the over-range measurement was decreased when the lactose was mechanofusion-processed, especially with additive. For Pharmatose $325 \mathrm{M}, 44 \%$ of measurements were over-range with intact lactose. The ratio decreased to $12 \%$ when the lactose was mechanofusion-processed without additive. When Pharmatose $325 \mathrm{M}$ was mechanofusion-processed with additive, all measurements were within the measurable range. For Lactohale 100, the adhesive force range was apparently narrowed when the mechanofusion process was conducted with additive.

Microscopic Observation of the Drug Particles Adhering to the Carrier Lactose Adhesion of $\mathrm{CBB}$ particles to the surface of the lactose carriers was observed with a digital microscope which could detect color (Fig. 7). The microscopic photographs of intact lactose or lactose mechanofusion-processed without additive showed that agglomerates or large particles of $\mathrm{CBB}$ adhered to the lactose surface. On the other hand, fewer agglomerates of $\mathrm{CBB}$ particles adhered to the surface of lactose mechanofusion-processed with additive. From these observations, it was suggested that both intact lactose and lactose mechanofusion-processed without additive tend to form agglomerates of the CBB particles adhering to the narrow highly adhesive sites.

\section{Discussion}

Similar alterations of inhalation properties induced by mechanofusion with Mg-St were observed for DPI formulations with different drug compounds or different particle size of carriers. This suggested the wide applicability of mechanofusion with $\mathrm{Mg}$-St for the improvement of the inhalation properties of DPI formulations (Fig. 1). On the contrary, Begat et al. suggested that lactose mechanofusionprocessed with $\mathrm{Mg}$-St decreased FPF in their work ${ }^{28)}$ where size of lactose carrier was relatively small (less than $10 \mu \mathrm{m}$ ), high drug/carrier ratio in powder formulation (1/1) and a different model drug was applied. In addition, even in our present study, dependence of inhalation profile on carrier property was varied as described in 'Results.' Therefore, although wide application of mechanofusion with lubricants is expected as mentioned above, there still are limitations with respect to drug property and need for further optimization of property of carrier particles and mixing conditions. The further examination focusing on those exceptions would be of 
(a)

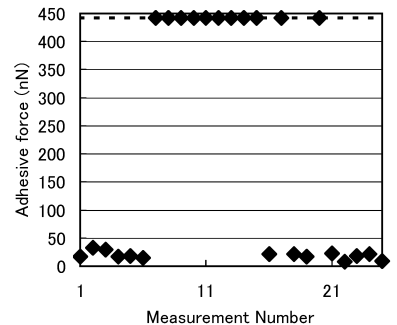

(e)

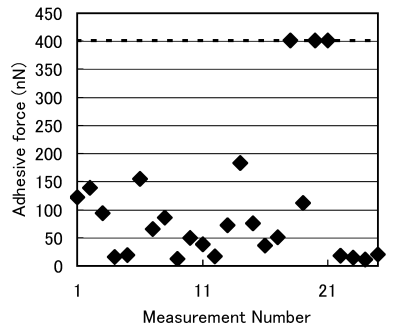

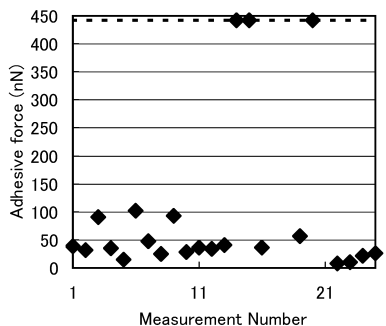

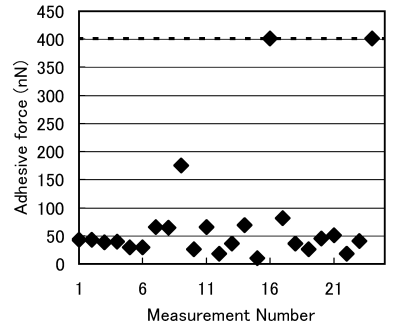

(c)

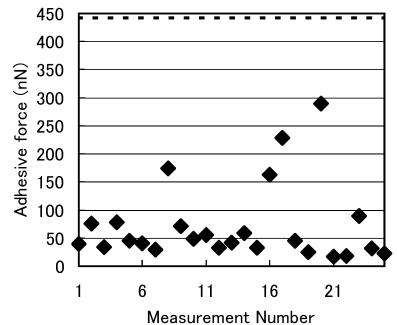

(g)

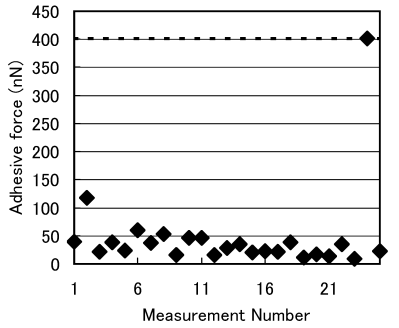

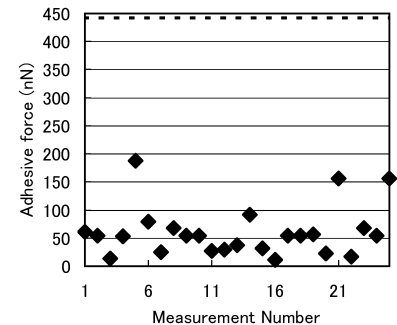

(h)

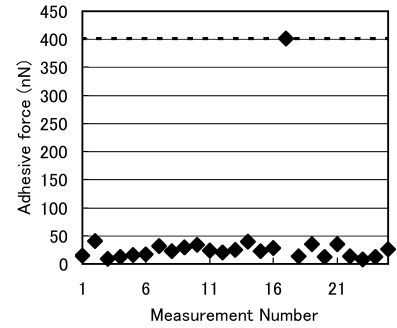

Fig. 6. Adhesive Force between TNRA and Lactose Carrier Surface Measured with PAF-300N

Each figure on the upper row shows the adhesive force between TNRA and intact Pharmatose 325M (a) or Pharmatose 325M mechanofusion-processed without additive (b), with sucrose stearate (c) and with Mg-St (d). Each figure on the lower row shows the adhesive force between TNRA and intact Lactohale 100 (e) or Lactohale 100 mechanofusionprocessed without additive (f), with sucrose stearate (g) and with Mg-St (h).
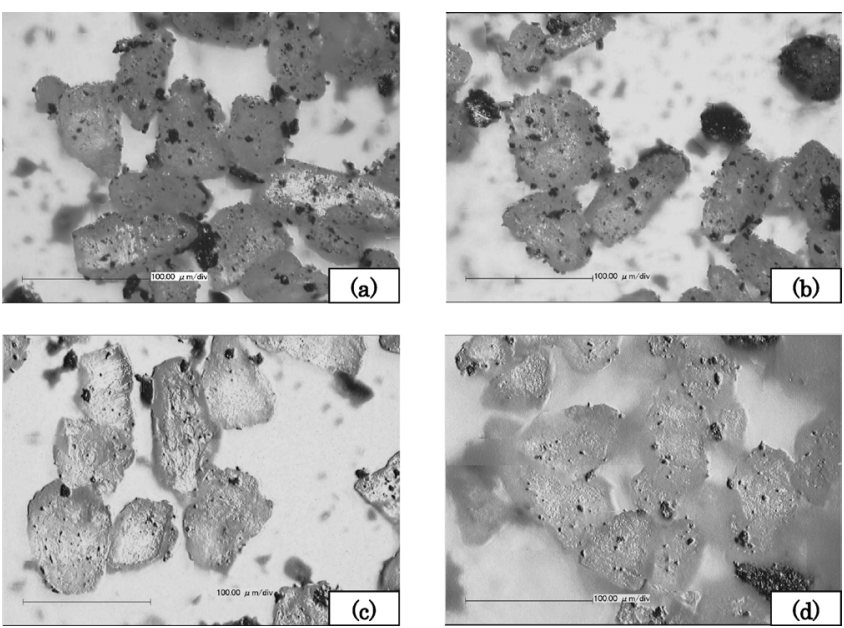

Fig. 7. Microscopic Images of Drug Particle Adhesion to Carrier Lactose Particle

CBB was mixed with intact Pharmatose $325 \mathrm{M}$ (a), Pharmatose $325 \mathrm{M}$ mechanofusion-processed without additive (b), with sucrose stearate (c), with Mg-St (d).

help to clarify these influencing factors on the drug-lactose interaction. The ACI deposition profile suggested that the drug particles were dispersed easily to show a high FPF value when they were formulated with lactose mechanofusion-processed with Mg-St (Fig. 2). Particle size distribution measurement implied that the higher FPF by mechanofusion with Mg-St was caused by higher dispersibility of the drug particles from the formulated powder mixture.

Both easier separation of the drug particles from the carrier and the formation of fewer agglomerates of the drug particles have been suggested to increase the dispersibility of the drug particles. ${ }^{10,37)}$ Based on the strategy of increasing FPF by decreasing the drug-carrier interaction, various techniques have been studied in order to improve the inhalation properties of DPI. ${ }^{22,38-42)}$
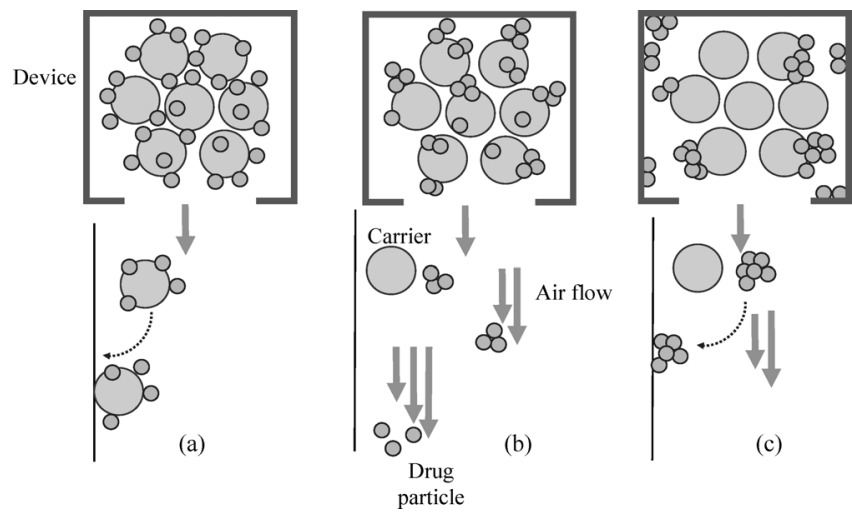

Fig. 8. Effect of Adhesive Force between Drug and Carrier Particles on the Inhalation Property of DPI Containing Carrier Particles

Small circles and large circles represent the drug and carrier lactose particles, respectively. Arrows show the direction of the inhalation air stream and boxes are simplified figures of the inhaler devices. Drug particles adhere to the carrier surface strongly and do not detach from it in the air stream (a). Interaction between the drug and carrier particles is so weak that the drug particles become agglomerated or adhere to the device wall (c). Interaction between the drug and carrier particles is at the appropriate level and the drug particles maintain a dispersed condition and easily detach from the carrier in the air stream (b).

A schematic diagram of the estimated relation between the drug-carrier interaction and the inhalation properties is illustrated in Fig. 8. When the interaction was too strong, the drug particles couldn't be released from the carrier into the air stream and would be deposited on the upper part of the trachea (Fig. 8a), ${ }^{43}$ even though, the drug particles would adhere to the carrier surface and form fewer agglomerates. On the contrary, when the interaction is too weak, the drug particles easily become agglomerated or adhere to the device wall. Agglomerates deposited on the upper part of the trachea and drug particles adhering to the device wall would not be inhaled deep into the lungs (Fig. 8c) resulting in low FPF. Therefore, it follows that intermediate interaction that keeps the drug particles in a well dispersed condition and easily re- 
leases the drug particles into the inhalation air stream would be advantageous to achieve high FPF (Fig. 8b).

In this study, the ACI deposition profile and particle size distribution data seemed to be compatible with the schematic diagram that the weaker drug-carrier interaction caused the higher FPF (as the comparison of Figs. 8a and b). To approach the mechanism, $\gamma_{\mathrm{s}}^{\mathrm{D}}$, the contact area and adhesive force were measured.

The $\gamma_{\mathrm{s}}^{\mathrm{D}}$ was increased by mechanofusion process but FPF decreased when mechanofusion was conducted without additive. However, in the comparison of three types of mechanofusion-processed lactose, increase of $\gamma_{\mathrm{s}}^{\mathrm{D}}$ was correlated with the FPF increase (Table 3). As the major part of $\gamma_{\mathrm{s}}^{\mathrm{D}}$ was constituted of van der Waals forces that were reported to make the predominant contribution to the total surface energy of dry aerosol particles, ${ }^{30)}$ mechanofusion was estimated to increase the interaction between the carrier and the drug particles. In the case of mechanofusion without additive, the decrease of FPF and detachment of drug particles from lactose with increase of $\gamma_{\mathrm{s}}^{\mathrm{D}}$ conformed to the mechanism shown in Fig. 8a. However, in the case of mechanofusion with Mg-St, FPF increased with the decrease of the actual interaction between drug and lactose while $\gamma_{\mathrm{s}}^{\mathrm{D}}$ increased. Therefore, the estimations of drug-lactose interaction based on ACI deposition profile and $\gamma_{\mathrm{s}}^{\mathrm{D}}$ were incompatible. Then it was implied that some factor other than surface energy affected the interaction between drug and lactose mechanofusion-processed.

The contact area is one of the determining factors of the interaction between the two particles. The lactose mechanofusion-processed with $\mathrm{Mg}$-St constantly showed high FPF over the wide range of surface area. Therefore, in this study, the contact area didn't seem to affect the interaction between the drug and lactose particles.

The repetitive adhesive force measurement suggested that mechanofusion process with additive homogenized the adhesive area distribution over the lactose surface (Fig. 6). The frequency of the over-range measurement of the adhesive force could be considered to reflect the distribution of adhesive areas. If a highly adhesive area and a less adhesive area localized separately on a carrier particle surface, the adhesive force would deviate widely. On the contrary, if the adhesiveness was homogeneous over the surface, the adhesive force would be in a narrow range. Therefore, the mechanofusion process, especially with additive, was suggested to homogenize the surface adhesiveness of the carrier lactose particles. It was reported that there were high energy binding sites i.e., adhering areas, on the carrier surface..$^{38,44,45)}$ Drug particles attached to those sites are considered not to be detached by the air flow. Therefore, fine additives are often utilized to cover the high energy binding sites and increase FPF. In the mechanofusion process, additives, such as $\mathrm{Mg}-\mathrm{St}$, also seemed to cover the high energy binding sites. Although the covering of high energy sites was expected, the following mechanofusion process with sheering and compressing spread the additive over the lactose surface and led to the additive having a brand-new surface with high energy. Therefore, mechanofusion with additive seemed to homogenize the surface adhesiveness through these steps.

Based on the two considerations above, i.e., the incompatibility between $\gamma_{\mathrm{s}}^{\mathrm{D}}$ and drug-lactose interaction change and

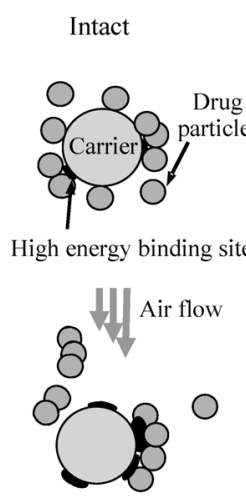

(a)
Mechanofusion processed
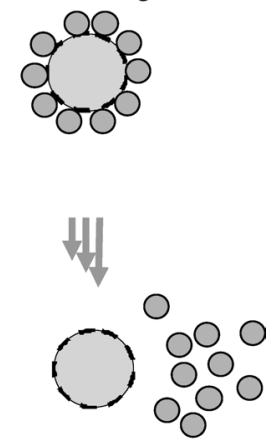

(b) with Mg-St

Fig. 9. Illustration of Inhalation Property Improvement Mechanism with Mechanofusion Process with Mg-St

Small circles and large circles represent the drug and carrier lactose particles, respectively. Arrows show the direction of the inhalation air stream. Intact lactose (a) and lactose mechanofusion-processed with $\mathrm{Mg}-\mathrm{St}(\mathrm{b})$.

homogenization of surface adhesiveness, we propose the hypothesis as follows: mechanofusion with $\mathrm{Mg}-\mathrm{St}$ spreads "extremely small high energy sites" homogeneously over the particle surface and the uniform adhesive surface of the carrier generated by mechanofusion with additive is advantageous to the achievement of a high FPF value. Our hypothesis of the mechanism whereby FPF increased with $\mathrm{Mg}$-St is illustrated in Fig. 9. Intact lactose would have highly adhesive "high energy binding areas" localized on the surface (Fig. 9a). These "high energy binding areas" could be considered as clusters of the "extremely small high energy sites." The drug particles would strongly adhere to the localized binding sites too strongly to be released by the air flow. On the other hand, when lactose was mechanofusion processed with additive, especially with $\mathrm{Mg}-\mathrm{St}$, "extremely small high energy sites" would be spread over the lactose surface homogeneously as $\mathrm{Mg}$-St got spread over the lactose surface. At the same time there would be fewer localized binding areas (clusters) as Mg-St adhere to those high energy binding sites prior to the lower energy sites. This alteration of the surface adhesiveness condition is considered to meet the requirements of the most appropriate interaction described above (Fig. 8). Namely, the drug particles were maintained in a well dispersed condition with fewer agglomerates, and could be easily separated or dispersed in the air stream (Fig. 8b). We consider that the number of "extremely small high energy sites" was increased by the compressing and shearing pressure through mechanofusion process, being reflected to the increase of $\gamma_{\mathrm{s}}^{\mathrm{D}}$, as IGC selectively detects the small high energy sites where the probe molecules could access. On the other hand, the homogenization by mechanofusion process could reduce the clusters of those areas, i.e. highly adhesive binding sites which were detected by PAF-300N. For the construction of the model in Fig. 9, $\gamma_{\mathrm{s}}^{\mathrm{D}}$ provided the information that mechanofusion process increased the surface energy that would contribute to keep the drug particles well dispersed; in contrast, direct measurement of adhesive force provided the information that the surface adhesiveness was homogenized through mechanofusion process. These two examinations seem to explain the mechanism of FPF increase by dispersibility increase that goes along with increase of surface energy. As there are various factors to be considered to inter- 
pret the inhalation properties of DPI formulations, further investigation should be conducted to study how the homogenization of the surface adhesiveness attributes to DPI performance. The variations of the mechanofusion conditions with different surface energy outcomes would be valuable in supporting the schematic mechanism of FPF increase by mechanofusion with Mg-St.

Throughout this study, it was suggested that utilizing a combination of different methods for surface characterization was valuable. IGC would be one of the best techniques to evaluate the highly adhesive sites for the probes and selectively detects the changes of high energy sites with high sensitivity. Thus, IGC could be utilized to evaluate the difference between the surface properties of carrier. In contrast, the direct separation method also measures the adhesive force of a specific site where the probe has contact and provides the information of the interaction of drug particles and the carrier surface. It differs from IGC on the following point: that the actual contact area would be reflected by using the lactose particle as a probe. In addition, the repetition of the measurement can give information regarding the whole surface of the sample particle, just as the scanning AFM direct measurement method also gives valuable information. ${ }^{46}$

\section{Conclusion}

The mechanofusion with $\mathrm{Mg}-\mathrm{St}$ was suggested to be widely applicable to the DPI formulations. And it was implied that mechanofusion with Mg-St increased the FPF of DPI formulation by both appropriately increasing the surface energy and homogenizing the surface adhesiveness over the carrier surface. The combination of direct adhesive force measurement, inhalation profile evaluation and surface energy measurement seemed to be of great help in DPI formulation development.

Acknowledgements The authors wish to thank Friesland Foods Domo and DMV for providing lactose. The authors are grateful to Hosokawa Micron Corporation for their technical assistance with the mechanofusion process. The authors wish to express their appreciation to Dr. Yasuhiro Shimada of Nano Seeds Corporation for his great help and advice regarding adhesive force measurement. Lastly, the authors wish to thank Dr. Yuji Kasuya of Daiichi Sankyo Co., Ltd. for his great help in preparation of the manuscript.

\section{References}

1) O’Hara P., Hickey A. J., Pharm. Res., 17, 955-961 (2000).

2) Sharma R., Saxena D., Dwivedi A. K., Misra A., Pharm. Res., 18, 1405-1410 (2001).

3) Smith D. J., Gambone L. M., Tarara T., Meays D. R., Dellamary L. A., Woods C. M., Weers J., Pharm. Res., 18, 1556-1561 (2001).

4) Tronde A., Nordén B., Marchner H., Wendel A. K., Lennernäs H., Bengtsson U. H., J. Pharm. Sci., 92, 1216-1233 (2003).

5) Bosquillon C., Preat V., Vanbever R., J. Controlled Release, 96, 233 244 (2004).

6) Yamamoto H., Kurashime H., Katagiri D., Yang M., Takeuchi H., Kawashima Y., Yokoyama T., Tsujimoto H., Yakuzaigaku, 64, 245253 (2004).

7) Owens D. R., Grimley J., Kirkpatrick P., Nat. Rev. Drug Discov., 5, $371-372$ (2006)

8) Fisher F. X., Pharm. Technol. Int., 1, 16-18 (1989).
9) Braun M. A., Oschmann R., Schmidt P. C., Int. J. Pharm., 135, 53-62 (1996).

10) Kawashima Y., Serigano T., Hino T., Yamamoto H., Takeuchi H., Int J. Pharm., 172, 179-188 (1998).

11) Chew N. Y., Chan H. K., Pharm. Res., 16, 1098-1103 (1999).

12) Chew N. Y., Bagster D. F., Chan H. K., Int. J. Pharm., 206, 75-83 (2000).

13) Islam N., Stewart P., Larson I., Hartley P., J. Pharm. Sci., 93, 1030 1038 (2004).

14) Steckel H., Markefka P., TeWierik H., Kammelar R., Eur. J. Pharm. Biopharm., 57, 495-505 (2004)

15) Ganderton D., Kassem N. M., International Patent No. WO 91/11179 (1991).

16) Staniforth J. N., International Patent No. WO 96/23485 (1996).

17) Podczeck F., Int. J. Pharm., 160, 119-130 (1998).

18) Podczeck F., Aerosol Sci. Technol., 31, 301-321 (1999).

19) Zeng X. M., Martin A. P., Marriott C., Pritchard J., Int. J. Pharm., 200 93-106 (2000).

20) Zeng X. M., Martin G. P., Marriott C., Pritchard J., J. Pharm. Sci., 90 1424-1434 (2001).

21) Jones M. D., Price R., Pharm. Res., V23, 1665-1674 (2006).

22) Schiavone H., Palakodaty S., Clark A., York P., Tzannis S. T., Int. J. Pharm., 281, 55-66 (2004).

23) Tong H. H. Y., Shekunov B. Y., York P., Chow A. H. L., J. Pharm. Sci. 95, 228-233 (2006).

24) Byron P. R., Peart J., Staniforth J. N., Pharm. Res., 14, 698-705 (1997)

25) Crowder T. M., Rosati J. A., Schroeter J. D., Hickey A. J., Martonen T B., Pharm. Res., 19, 239-245 (2002).

26) Murtomaa M., Mellin V., Harjunen P., Lankinen T., Laine E., Lehto V. P., Int. J. Pharm., 282, 107-114 (2004).

27) Kumon M., Suzuki M., Kusai A., Yonemochi E., Terada K., Chem. Pharm. Bull., 54, 1508-1514 (2006).

28) Begat P., Price R., Harris H., Morton D. A. V., Staniforth J. N., KONA 23, 109-121 (2005).

29) Manfred K., Rudi M. W., U.S. Patent 7186401 (2003).

30) Shekunov B. Y., Feeley J. C., Chow A. H. L., Tong H. Y. Y., York P., J. Aerosol Sci., 34, 553-568 (2003).

31) Davies M., Brindley A., Chen X., Marlow M., Doughty S. W., Shrubb I., Roberts C. J., Pharm. Res., 22, 1158-1166 (2005).

32) Schultz J., Lavielle L., Martin C., J. Adh., 23, 45-60 (1987).

33) Grimsey I. M., Feeley J. C., York P., J. Pharm. Sci., 91, 571-583 (2002).

34) Nardin M., Papirer E., J. Colloid Interface Sci., 137, 534-545 (1990).

35) Shimada Y., Yonezawa Y., Sunada H., J. Pharm. Sci., 92, 560-568 (2003).

36) Sunada H., Yakuzaigaku, 66, 2-11 (2006).

37) Staniforth J. N., "Proceedings from Respiratory Drug Delivery V," Phoenix, AZ, 1996, pp. 65-74.

38) Zeng X. M., Pandhal K. H., Martin G. P., Int. J. Pharm., 197, 41-52 (2000).

39) Zeng X. M., Martin G. P., Marriott C., Pritchard J., J. Pharm. Pharmacol., 52, 1211-1221 (2000).

40) Ikegami K., Kawashima Y., Takeuchi H., Yamamoto H., Isshiki N., Momose D., Ouchi K., Pharm. Res., 19, 1439-1445 (2002).

41) Onoue M., Endo K., Japan Patent, P2002-284703A, (2002).

42) Larhrib H., Martin G. P., Marriott C., Prime D., Int. J. Pharm., 257, 283-296 (2003)

43) Begat P., Morton D. A., Staniforth J. N., Price R., Pharm. Res., 21, 1826 - 1833 (2004)

44) Zeng X. M., Martin G. P., Tee S. K., Ghoush A. A., Marriott C., Int. J. Pharm., 182, 133-144 (1999).

45) Young P. M., Edge S., Traini D., Jones M. D., Price R., El-Sabawi D., Urry C., Smith C., Int. J. Pharm., 296, 26-33 (2005).

46) Zhang J., Ebbens S., Chen X., Jin Z., Luk S., Madden C., Patel N., Roberts C. J., Pharm. Res., V23, $401-407$ (2006). 